\title{
Sonceal Nafion-Induced Reduction of Manganese and its Impact on the Electrocatalytic Properties of a Highly Active MnFeNi Oxide for Bifunctional Oxygen Conversion**
}

\author{
Dulce M. Morales, ${ }^{*[a]}$ Javier Villalobos, ${ }^{[a]}$ Mariya A. Kazakova ${ }^{[b]}$ Jie Xiao, ${ }^{[c]}$ and Marcel Risch ${ }^{*[a]}$
}

Electrocatalysts for bifunctional oxygen reduction (ORR) and oxygen evolution reactions (OER) are commonly studied under hydrodynamic conditions, rendering the use of binders necessary to ensure the mechanical stability of the electrode films. The presence of a binder, however, may influence the properties of the materials under examination to an unknown extent. Herein, we investigate the impact of Nafion on a highly active ORR/OER catalyst consisting of $\mathrm{MnFeNi}$ oxide nanoparticles supported on multi-walled carbon nanotubes. Electrochemical studies revealed that, in addition to enhancing the mechanical stability and particle connectivity, Nafion poses a major impact on the ORR selectivity, which correlates with a decrease in the valence state of $\mathrm{Mn}$ according to X-ray absorption spectroscopy. These findings call for awareness regarding the use of electrode additives, since in some cases the extent of their impact on the properties of electrode films cannot be regarded as negligible.

Electrocatalytic oxygen conversion-comprising the oxygen evolution (OER) and the oxygen reduction reaction (ORR)-is considered a major obstacle for the commercialization of green, regenerative energy conversion technologies, for example, reversible fuel cells or rechargeable metal-air batteries, since both reactions suffer from sluggish kinetics. ${ }^{[1]}$ Hence, the design of low-cost, high-performance bifunctional ORR/OER electrocatalysts (BOEs) is essential to increase the efficiency of these

[a] Dr. D. M. Morales, J. Villalobos, Dr. M. Risch

Nachwuchsgruppe Gestaltung des Sauerstoffentwicklungsmechanismus

Helmholtz-Zentrum Berlin für Materialien und Energie $\mathrm{GmbH}$

Hahn-Meitner-Platz 1, 14109 Berlin, Germany

E-mail: dulce.morales_hernandez@helmholtz-berlin.de marcel.risch@helmholtz-berlin.de

[b] Dr. M. A. Kazakova

Boreskov Institute of Catalysis, SB RAS

Lavrentieva 5, 630090 Novosibirsk, Russia

[c] Dr. J. Xiao

Department of Highly Sensitive X-ray Spectroscopy

Helmholtz-Zentrum Berlin für Materialien und Energie $\mathrm{GmbH}$

Albert-Einstein-Straße 15, 12489 Berlin, Germany

[**] A previous version of this manuscript has been deposited on preprint servers (DOls: https://arxiv.org/abs/2105.13888, https://doi.org/10.26434/ chemrxiv.14696463.v1)

Supporting information for this article is available on the WWW under https://doi.org/10.1002/celc.202100744

An invited contribution to a Special Collection on Bifunctional

Electrocatalysis

(c) 2021 The Authors. ChemElectroChem published by Wiley-VCH GmbH. This is an open access article under the terms of the Creative Commons Attribution License, which permits use, distribution and reproduction in any medium, provided the original work is properly cited. devices. ${ }^{[2]}$ Investigation of BOEs is typically conducted under hydrodynamic conditions using rotating disk electrode (RDE) setups or electrochemical flow cells..$^{[3,4,5]}$ It is thus necessary that the mechanical stability of the investigated electrode film is sufficiently high to endure the experimental conditions required for the evaluation of its catalytic properties. This can be achieved by means of a binder, which not only aids in preventing catalyst detachment, but also in improving the electric contact between catalyst particles and electrode substrate, lowering thus the resistance of the electrode film. ${ }^{[6,7]}$ Depending on the binder and its concentration, additional effects on various properties of electrode films have been reported, including ionic conductivity, hydrophobicity, mass transport, and accessibility to the reaction sites, often leading to an improvement of the catalytic performance. ${ }^{[8-10]}$

A popular compound used as a binder is Nafion, an ionconducting ionomer comprising hydrophilic, sulfonic-terminated side chains, and built upon copolymerization of tetrafluoroethylene and perfluorinated vinyl ether monomers. ${ }^{[11]}$ This binder has proved successful in improving the mechanical stability of electrode films, and thus it has been recommended for benchmark electrode preparation protocols. ${ }^{[12,13]}$ It is reported that Nafion may induce decreases in overpotential attributed to an increased electrical conductivity and facilitated mass transport, ${ }^{[6]}$ though depending largely on the electrode composition, ${ }^{[8,12,14]}$ and not impacting otherwise the catalytic activity of, for instance, $\operatorname{IrO}_{2}{ }^{[10,12]} \mathrm{Pt} / \mathrm{C}^{[6,15]}$ or $\mathrm{Pt}-\mathrm{Sn} / \mathrm{C}^{[15]}$ However, the nature of additional effects observed with diverse materials remains unclear. It has been speculated that Nafion impacts the intrinsic catalytic properties of $\mathrm{Pt}$, attributed to the specific adsorption of sulfonate groups on the catalyst surface. ${ }^{[8]}$ Moreover, it was recently reported that the OER activity trend exhibited by $\mathrm{Mn}$ oxides of various crystal structures was different in the presence of Nafion than in its absence, speculatively due to a binder-induced chemical change of the surface of these materials. ${ }^{[16]}$ Furthermore, the acidic nature of Nafion may also lead to corrosion and catalyst dissolution in the case of materials that are not chemically stable in low $\mathrm{pH}$ media, ${ }^{[5]}$ impacting further the apparent activity of the investigated catalyst films. Hence, understanding the influence of Nafion on the catalytic properties of materials under investigation results crucial for studies that aim to elucidate their intrinsic properties.

As a case study, we investigate multiphase $\mathrm{Mn}, \mathrm{Fe}$ and $\mathrm{Ni}$ oxide nanoparticles $\left(\mathrm{Mn}_{0.51} \mathrm{Fe}_{0.14} \mathrm{Ni}_{0.35}\right.$ metal composition and 14.4 wt $\%$ total metal loading) supported on oxidized multiwalled carbon nanotubes, hereafter denoted MnFeNiOx, which 
was recently proposed as a high-performance $\mathrm{BOE} .{ }^{[17]}$ According to a previous TEM study, the nanoparticles are located inside and outside the walls of the nanotubes in a 1.25:1 ratio, with an average particle size of $3.6 \pm 1.2 \mathrm{~nm}$ and $10.5 \pm 5.9 \mathrm{~nm} \cdot{ }^{[17]}$ HAADF STEM and EDX elemental analysis (Figure S1) indicates that each particle has its own individual composition. Yet, the composition averaged to $50 \mathrm{~nm}$ was $\mathrm{Mn}_{0.51} \mathrm{Fe}_{0.18} \mathrm{Ni}_{0.31}$, which is in fair agreement with previous XRF analysis. ${ }^{[17]}$ Additionally, the elemental maps shown in Figure $\mathrm{S} 1$ suggest that the three metals are likely to be exposed to Nafion during the treatments described in the following. Catalyst inks (MnFeNiOx dispersed in a 1:1 water-ethanol mixture) were deposited onto glassy carbon (GC) RDEs in the presence or absence of 2 vol\% Nafion solution ( $\sim 5 \%$ Nafion in a mixture of alcohols) in order to observe its impact on the electrocatalytic properties of the obtained catalyst films. The binder-containing and binder-free films are hereafter denoted MnFeNiOx-Nafion/GC and MnFe$\mathrm{NiOx} / \mathrm{GC}$, respectively. Linear sweep voltammograms (LSVs) of MnFeNiOx/GC and MnFeNiOx-Nafion/GC were recorded in triplicate in the ORR and OER potential regions. The individual measurements and their averages are shown in Figure S2 and Figure $1 \mathrm{a}$, respectively. To facilitate the comparison, we determined the potential at which disk current densities of $+10 \mathrm{~mA} \mathrm{~cm}^{-2}\left(E_{O E R}\right)$ and $-1 \mathrm{mAcm}^{-2}\left(E_{O R R}\right)$ were attained for the two samples as activity metrics ${ }^{[4,18]}$ (Table S1). The obtained $E_{O E R}$ values were $1.547 \pm 0.006$ and $1.538 \pm 0.007 \mathrm{~V}$ vs RHE for MnFeNiOx/GC and MnFeNiOx-Nafion/GC, respectively, indicating only a slight increase in the apparent OER activity of the electrodes upon addition of Nafion, which could be explained by an improved contact between the conductive MnFeNiOx powder and the GC substrate, ${ }^{[10]}$ while differences in mass transport and availability of the active sites may also play a role. Similarly in the case of the ORR, MnFeNiOx-Nafion/GC displayed an $E_{O R R}$ value of $0.788 \pm 0.003 \mathrm{~V}$ vs $\mathrm{RHE}$, while for $\mathrm{MnFeNiOx} / \mathrm{GC}$ $E_{O R R}$ was $0.776 \pm 0.009 \mathrm{~V}$ vs $\mathrm{RHE}$, with a comparatively higher reproducibility in the case of the former as shown in Figure S2. Yet, a more substantial difference in the recorded ORR currents was observed in the kinetic-diffusion mixed control region, which could be related to differences in ORR selectivity. To investigate this, LSVs were recorded at different electrode rotation rates, and the number of electrons transferred during the ORR ( $n$ ) was determined via the Koutecky-Levich (K-L) analysis. ${ }^{[19,20]}$ The analysis was conducted from three independent sets of measurements (Figure S3) extracting data at 0.55, $0.60,0.65$ and $0.70 \mathrm{~V}$ vs RHE (Figure S4), and displaying high reproducibility in the investigated potential range (Table S2). Average LSVs and K-L plots obtained at $0.65 \mathrm{~V}$ vs RHE are displayed in Figure $1 \mathrm{~b}$ and $1 \mathrm{c}$, respectively. For MnFeNiOx/GC, $n$ had a value of 2.3 , which translates into a pathway favoring the formation of peroxide species $(n=2)$, according to Equation (1) ${ }^{[20]}$ Interestingly, for the Nafion-containing sample $n$ was 3.8, indicating that the direct reduction of $\mathrm{O}_{2}$ to $\mathrm{OH}^{-}$is favored [Eq. (2)], ${ }^{[20]}$ which agrees with an earlier study conducted by rotating ring disk electrode voltammetry. ${ }^{[1]]}$ These results indicate that the presence of Nafion leads to a major improvement of the ORR selectivity of the trimetallic catalyst.
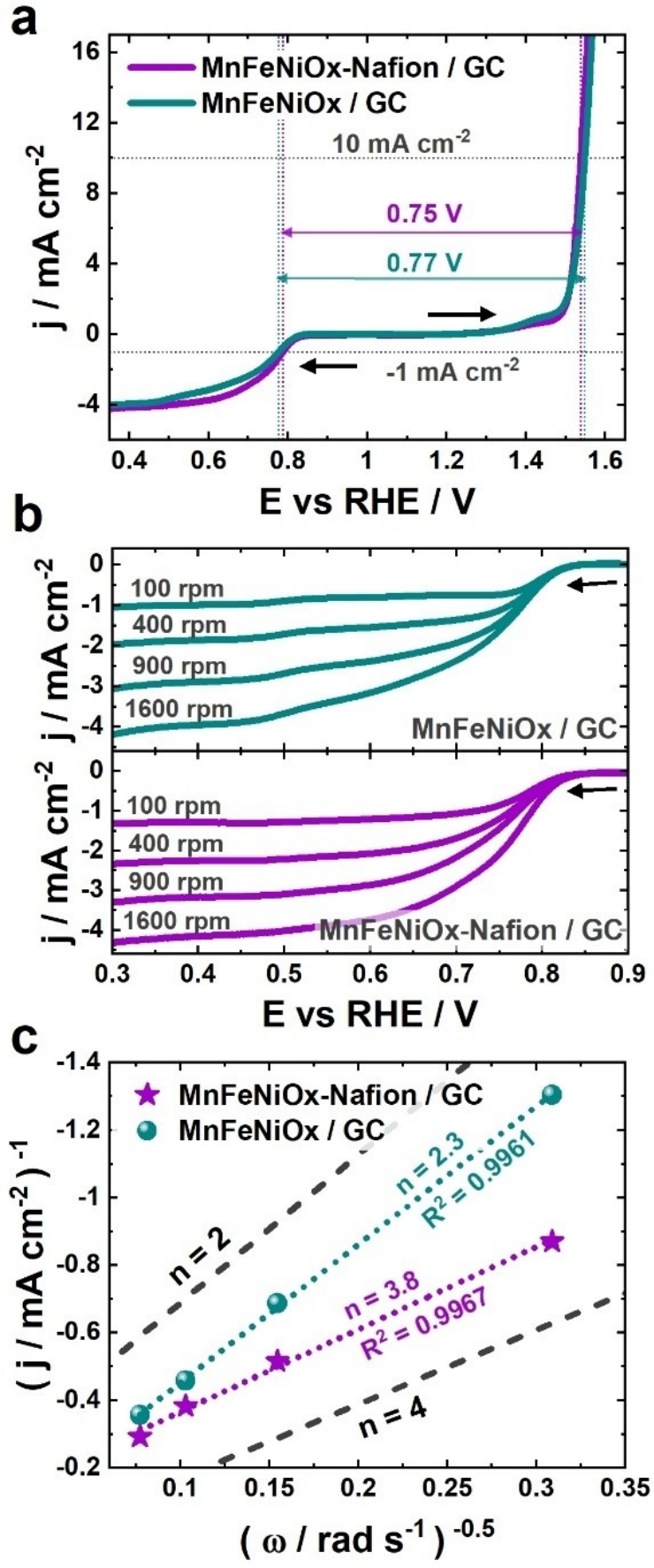

Figure 1. iR -drop-compensated LSVs corresponding to MnFeNiOx deposited onto GC-RDEs in the presence (purple) and in the absence (teal) of Nafion with a scan rate of $5 \mathrm{mV} \mathrm{s}^{-1}$ (a) at $1600 \mathrm{rpm}$ electrode rotation in the OER and ORR potential regions, (b) at different electrode rotation rates in the ORR potential region, and (c) their corresponding Koutecky-Levich plots obtained at $0.65 \mathrm{~V}$ vs RHE. Simulated plots corresponding to the transfer of 2 and 4 electrons are shown for guidance, and were determined considering $D=1.9 \times 10^{-5} \mathrm{~cm}^{2} \mathrm{~s}^{-1}, v=1.1 \times 10^{-2} \mathrm{~cm}^{2} \mathrm{~s}^{-1}$, and $C=1.2 \times 10^{-6} \mathrm{~mol} \mathrm{~cm}^{-3}$. ${ }^{[20]}$ All measurements were conducted in $\mathrm{O}_{2}$-saturated $0.1 \mathrm{M} \mathrm{NaOH}$ solution. Black arrows indicate the direction of the voltammetric scan.

$$
\begin{aligned}
& \mathrm{O}_{2}+\mathrm{H}_{2} \mathrm{O}+2 \mathrm{e}^{-} \rightarrow \mathrm{HO}_{2}^{-}+\mathrm{OH}^{-} \\
& \mathrm{O}_{2}+2 \mathrm{H}_{2} \mathrm{O}+4 \mathrm{e}^{-} \rightarrow 4 \mathrm{OH}^{-}
\end{aligned}
$$


MnFeNiOx was initially proposed as a two-component catalyst with FeNiOx (3:7 metal ratio) being the active site for the $\mathrm{OER}^{[21]}$ and $\mathrm{MnOx}$ being the key component that activates the catalyst towards the ORR. ${ }^{[1]}$ If this assumption is correct, and given that the presence of the binder led to a substantial enhancement of the ORR performance while barely influencing the OER activity, it can be hypothesized that the catalyst undergoes Nafion-induced chemical changes related to $\mathrm{Mn}$. Since correlations between $\mathrm{Mn}$ valence and ORR selectivity have been established, ${ }^{[22,23]}$ we resorted to X-ray absorption spectroscopy (XAS) for an in-depth investigation of our hypothesis.

XAS spectra were collected before (MnFeNiOx) and after (MnFeNiOx(Nafion)) treating the catalyst in a Nafion-containing water-ethanol solution by sonication for $15 \mathrm{~min}$ (see sample preparation protocol in Supporting Information). The spectra obtained in the $\mathrm{Ni}-\mathrm{L}_{3}, \mathrm{Fe}-\mathrm{L}_{3}$ and $\mathrm{Mn}-\mathrm{L}_{3}$ edges are shown in Figure $2 \mathrm{a}, 2 \mathrm{~b}$ and $2 \mathrm{c}$, respectively, displaying alongside the spectra of metal oxides of unmixed oxidation states for reference. While no substantial differences in the $\mathrm{Ni}-\mathrm{L}_{3}$ spectra were shown by MnFeNiOx and MnFeNiOx(Nafion) (Figure 2a), in the case of the $\mathrm{Fe}^{-} \mathrm{L}_{3}$ edge, a change in the background was observed at energies above $\sim 712 \mathrm{eV}$ (Figure $2 \mathrm{~b}$ ), attributed to the F-K edge absorption of Nafion's fluorine atoms (Figure $2 d) .{ }^{[24]}$ Yet, the prominent $F e-L_{3}$ peak did not feature any other visible change. In the case of the $M n-L_{3}$ energy region (Figure 2c), the spectra recorded for $\mathrm{MnFeNiOx}$ (teal) and MnFeNiOx(Nafion) (purple) displayed major differences. Earlier, XRD characterization revealed that MnFeNiOx consists of various mono and bimetallic oxide phases in a highly defective state. ${ }^{[17]}$ Thus, $\mathrm{Mn}$ in MnFeNiOx is expected to be present in a mixture of oxidation states, as observed in Figure $2 c$, with a larger prevalence of $\mathrm{Mn}^{2+}$ and $\mathrm{Mn}^{3+}$ surface species according to XPS analyses. ${ }^{[17]}$ The more intense feature centered at $\sim 641 \mathrm{eV}$ in the spectrum of MnFeNiOx(Nafion) compared to MnFeNiOx suggests that $\mathrm{Mn}$ became mainly present in oxidation state $2+$ upon exposure to Nafion-containing solution. Given that $\mathrm{Fe}$ and $\mathrm{Ni}$ spectra did not display any evident change that correlates with a variation of their oxidation states, it can be assumed that the majority of the binder-caused surface state changes that led to an improvement of the ORR selectivity are related to $\mathrm{Mn}$. This observation supports the hypothesis that $\mathrm{Mn}$ oxide is an essential component of the ORR active sites in MnFeNiOx.

The increase in the intensity of the spectral feature related to $\mathrm{Mn}^{2+}$ species could be attributed to a Nafion-induced chemical reduction, which has been observed previously with Mn-containing complexes. ${ }^{[25]}$ However, another plausible explanation is that the strongly acidic proton in the binder induces disproportionation of $\mathrm{Mn}^{3+}$, forming $\mathrm{Mn}^{2+}$ and $\mathrm{Mn}^{4+},{ }^{[26]}$ a

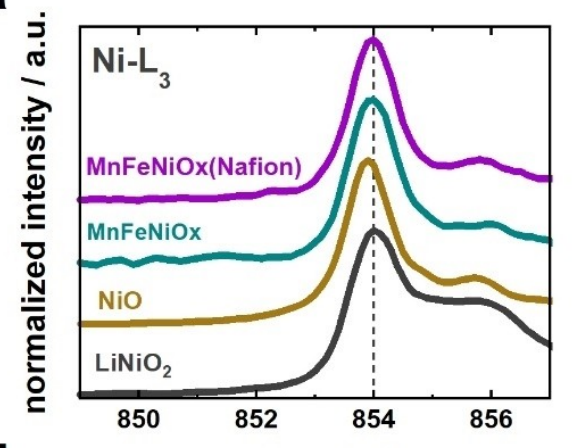

d

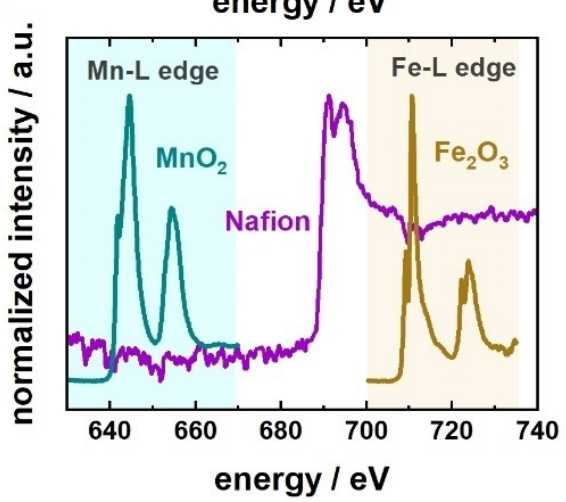

b
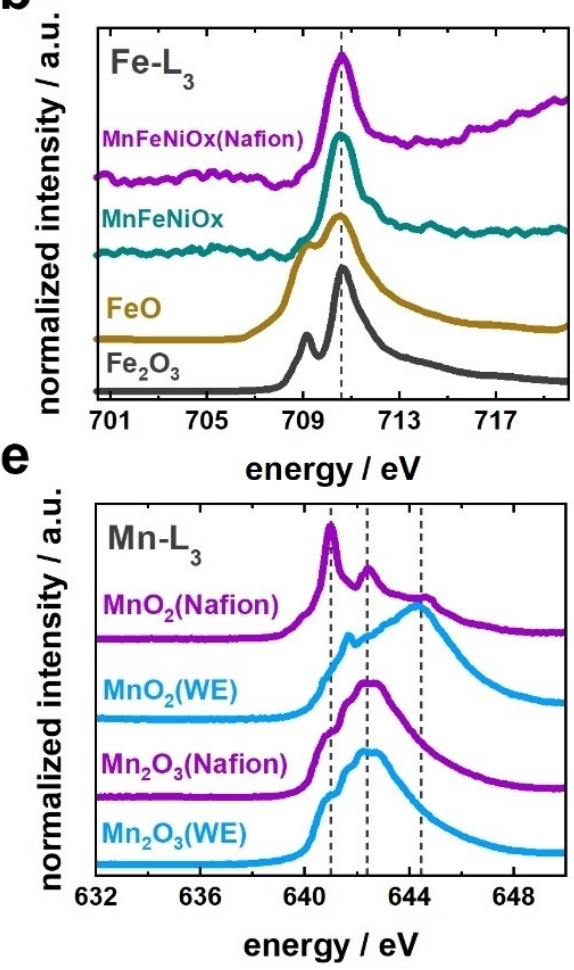

C

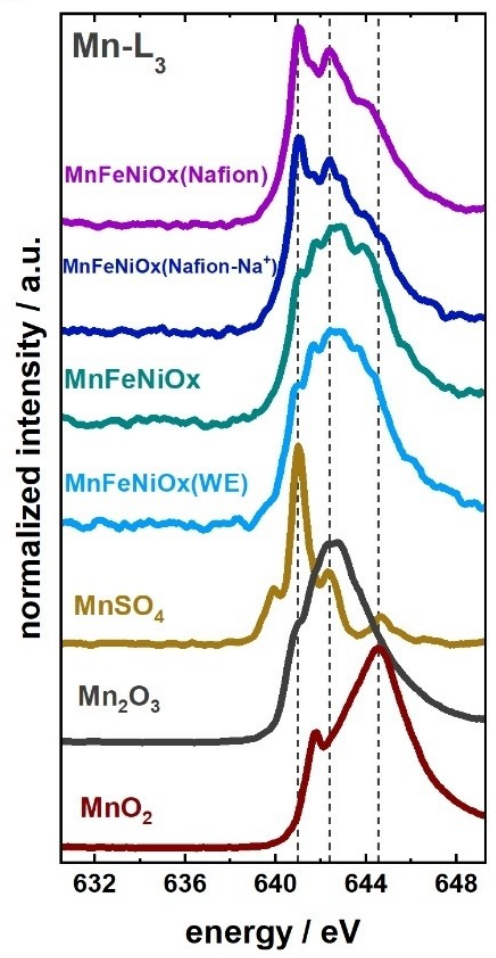

Figure 2. Normalized XAS spectra of MnFeNiOx recorded in total electron yield mode before and after treatment in water-ethanol mixtures without (WE) or with 2 vol\% binder solution (Nafion), recorded in the $L_{3}$ edge of a) Ni, b) Fe, and c) Mn, showing corresponding reference compounds; $c$ ) shows additionally the spectra of $\mathrm{MnFeNiOx}$ exposed to a WE solution containing cation-exchanged binder ( $\mathrm{Nafion}\left(\mathrm{Na}^{+}\right)$). d) XAS spectrum of $\mathrm{Nafion}$ in the energy region comprising both the $\mathrm{L}_{2}-\mathrm{L}_{3}$ edge of $\mathrm{Mn}$ and the $\mathrm{L}_{2}-\mathrm{L}_{3}$ edge of Fe; the spectra of $\mathrm{MnO}_{2}$ and $\mathrm{Fe}_{2} \mathrm{O}_{3}$ are shown for reference. e) XAS spectra of $\mathrm{MnO}_{2}$ and $\mathrm{Mn}_{2} \mathrm{O}_{3}$ recorded in the $\mathrm{L}_{3}$ edge of $\mathrm{Mn}$ after treatment in water-ethanol mixture in the abscence (WE) and in the presence of binder (Nafion). Spectra were offset for clarity and vertical dashed lines are included to guide the eye. 
and resulting in an apparent increase in $\mathrm{Mn}^{2+}$ species due to a variation in the ratio $\mathrm{Mn}^{2+}: \mathrm{Mn}^{3+}: \mathrm{Mn}^{4+}$. To further understand the origin of the change in $\mathrm{Mn}$ oxidation state, two control experiments were conducted with MnFeNiOx treated in (1) a water-ethanol mixture in the absence of Nafion (MnFeNiOx (WE)), and in (2) a solution containing cation-exchanged Nafion $\left(\mathrm{MnFeNiOx}\left(\right.\right.$ Nafion-Na $\left.{ }^{+}\right)$), namely, after having replaced the $\mathrm{H}^{+}$ at the sulfonate groups with $\mathrm{Na}^{+}$according to a previously reported procedure. ${ }^{[5]}$ The quality of the dispersions obtained when using cation-exchanged Nafion solution did not display any evident difference than that observed with Nafion in its acidic form. The obtained XAS spectra (Figure 2c) show that, while no substantial spectral difference was observed between untreated MnFeNiOx (teal) and MnFeNiOx(WE) (light blue), the changes observed with MnFeNiOx(Nafion) (purple) were displayed as well by $\mathrm{MnFeNiOx}\left(\mathrm{Nafion}-\mathrm{Na}^{+}\right.$) (dark blue), thereby suggesting that the acidic proton in the binder does not play a major role in the observed $\mathrm{Mn}$ valence changes.

We further investigated the effect of Nafion on the XAS features of $\mathrm{MnO}_{2}$ and $\mathrm{Mn}_{2} \mathrm{O}_{3}$, for which the corresponding powders were treated in a water-ethanol mixture in the absence $\left(\mathrm{Mn}_{X} \mathrm{O}_{Y}(\mathrm{WE})\right)$ or presence $\left(\mathrm{Mn}_{\mathrm{X}} \mathrm{O}_{Y}\right.$ (Nafion)) of the binder (Figure 2e). Interestingly, the peak assigned to $\mathrm{Mn}^{2+}(641 \mathrm{eV})$ was clearly seen with $\mathrm{MnO}_{2}\left(\mathrm{Mn}^{4+}\right)$ upon exposure to Nafion, whereas the spectra corresponding to $\mathrm{Mn}_{2} \mathrm{O}_{3}\left(\mathrm{Mn}^{3+}\right)$ did not display any substantial change, indicating that the valence changes are not related to disproportionation. We speculate that a strong chemical interaction between $\mathrm{Mn}^{4+}$ species in $\mathrm{MnO}_{2}$ and the electron donors in the binder takes place. Likely, this occurs similarly with $\mathrm{Mn}^{4+}$ species in MnFeNiOx, thus leading to an overall improvement in the ORR performance of the catalyst: on the one hand, the formation of $\mathrm{Mn}^{2+}$ species (from $\mathrm{Mn}^{4+}$ ) could favor the binding of ${ }^{*} \mathrm{OOH}$ intermediates according to recent DFT predictions, ${ }^{[27]}$ and on the other hand, the unaffected $\mathrm{Mn}^{3+}$ atoms provide $\mathrm{O}_{2}$ absorption sites ${ }^{[28]}$ and a $\mathrm{Mn}^{3+}: \mathrm{Mn}^{4+}$ ratio that facilitates the 4-electron transfer pathway. ${ }^{[23,29]}$

In summary, we investigated the influence of Nafion on the electrocatalytic properties of MnFeNiOx as a bifunctional ORR/ OER catalyst. Besides the advantageous, yet expected, improvement of electrode film properties (mechanical stability and particle contact), a major benefit on the ORR selectivity of MnFeNiOx was revealed: while binder-free MnFeNiOx displayed a preferred 2-electron transfer pathway, in the presence of Nafion the composite electrode exhibited the reduction of $\mathrm{O}_{2}$ to $\mathrm{OH}^{-}$predominantly via the transfer of 4 electrons. The impressive improvement in selectivity is attributed to a binderinduced decrease in the oxidation state of $\mathrm{Mn}$, as observed during XAS investigations, resulting in a more favorable $\mathrm{Mn}^{2+}$ : $\mathrm{Mn}^{3+}: \mathrm{Mn}^{4+}$ ratio, and confirming that $\mathrm{Mn}$ plays a major role in the ORR performance of the trimetallic catalyst. Control experiments on MnFeNiOx and commercial Mn oxides indicated, on the one hand, that the valence changes observed are neither related to the acidic nature of Nafion, nor to disproportionation, and on the other hand, that $\mathrm{Mn}^{4+}$ species are susceptible to chemical reduction in the presence of Nafion. Although further studies are still required to fully reveal the extent of the chemical changes, as well as the variety of materials that may be susceptible to them, it is clear that Nafion cannot always be regarded as inert, and that awareness on its use is required for investigations where the intrinsic activity of a catalytic material is the main focus of the work.

\section{Acknowledgments}

The authors are grateful to Dr. Ronny Golnak (HZB, Department of Highly Sensitive X-ray Spectroscopy), and Denis Antipin, Joaquín Morales-Santelices and Sepideh Madadkhani (HZB, Nachwuchsgruppe Gestaltung des Sauerstoffentwicklungsmechanismus) for their support during collection of XAS data, to Dr. Arcady V. Ishchenko (Boreskov Institute of Catalysis SB RAS) for performing TEM measurements, and to Christian Höhn (HZB, Institute for Solar Fuels) for preliminary XPS analyses. We thank HZB for the allocation of synchrotron radiation beamtime. This project has received funding from the European Research Council (ERC) under the European Union's Horizon 2020 research and innovation programme under grant agreement No 804092. M. A. K. acknowledges the support of the Ministry of Science and Higher Education of the Russian Federation within the state assignment for Boreskov Institute of Catalysis (project \# AAAA-A21-121011390054-1). Open access funding enabled and organized by Projekt DEAL.

\section{Conflict of Interest}

The authors declare no conflict of interest.

Keywords: bifunctional oxygen electrodes - Nafion - oxidation state $\cdot$ transition metals $\cdot \mathrm{X}$-ray absorption spectroscopy

[1] a) K. Zeng, X. Zheng, C. Li, J. Yan, J.-H. Tian, C. Jin, P. Strasser, R. Yang, Adv. Funct. Mater. 2020, 30, 2000503; b) W. T. Hong, M. Risch, K. A. Stoerzinger, A. Grimaud, J. Suntivich, Y. Shao-Horn, Energy Environ. Sci. 2015, 8, 1404

[2] a) S. Zhao, L. Yan, H. Luo, W. Mustain, H. Xu, Nano Energy 2018, 47, 172; b) X. Wu, C. Tang, Y. Cheng, X. Min, S. P. Jiang, S. Wang, Chem. Eur. J. 2020, 26, 3906.

[3] a) S.-J. Lee, S.-I. Pyun, S.-K. Lee, S.-J. L. Kang, Isr. J. Chem. 2008, 48, 215 ; b) I. Spanos, A. A. Auer, S. Neugebauer, X. Deng, H. Tüysüz, R. Schlögl, ACS Catal. 2017, 7, 3768.

[4] C. C. L. McCrory, S. Jung, J. C. Peters, T. F. Jaramillo, J. Am. Chem. Soc. 2013, 135, 16977.

[5] J. Suntivich, H. A. Gasteiger, N. Yabuuchi, Y. Shao-Horn, J. Electrochem. Soc. 2010, 157, B1263.

[6] E. Antolini, L. Giorgi, A. Pozio, E. Passalacqua, J. Power Sources 1999, 77 136.

[7] a) S. Cheng, H. Liu, B. E. Logan, Environ. Sci. Technol. 2006, 40, 364; b) R. Wang, L. Feng, W. Yang, Y. Zhang, Y. Zhang, W. Bai, B. Liu, W. Zhang, Y. Chuan, Z. Zheng et al., Nanoscale Res. Lett. 2017, 12, 575; c) A. Van Nguyen, C. Kuss, J. Electrochem. Soc. 2020, 167, 65501.

[8] T. Saito, M. D. Merrill, V. J. Watson, B. E. Logan, M. A. Hickner, Electrochim. Acta 2010, 55, 3398.

[9] X. Wang, Y. Feng, J. Liu, X. Shi, H. Lee, N. Li, N. Ren, Biosens. Bioelectron. 2010, 26, 946

[10] G.-F. Li, D. Yang, P.-Y. Abel Chuang, ACS Catal. 2018, 8, 11688.

[11] a) K. A. Mauritz, R. B. Moore, Chem. Rev. 2004, 104, 4535; b) H. Abroshan, H. Akbarzadeh, F. Taherkhani, G. Parsafar, Mol. Phys. 2011, 109, 709. 
[12] S. Jung, C. C. L. McCrory, I. M. Ferrer, J. C. Peters, T. F. Jaramillo, J. Mater. Chem. A 2016, 4, 3068.

[13] S. S. Kocha, K. Shinozaki, J. W. Zack, D. J. Myers, N. N. Kariuki, T. Nowicki, V. Stamenkovic, Y. Kang, D. Li, D. Papageorgopoulos, Electrocatalysis 2017, 8, 366.

[14] L. Köhler, M. Ebrahimizadeh Abrishami, V. Roddatis, J. Geppert, M. Risch, ChemSusChem 2017, 10, 4479.

[15] A. Manzo-Robledo, A.-C. Boucher, E. Pastor, N. Alonso-Vante, Fuel Cells 2002, 2, 109.

[16] T. Hayashi, N. Bonnet-Mercier, A. Yamaguchi, K. Suetsugu, R. Nakamura, R. Soc. Open Sci. 2019, 6, 190122.

[17] D. M. Morales, M. A. Kazakova, S. Dieckhöfer, A. G. Selyutin, G. V. Golubtsov, W. Schuhmann, J. Masa, Adv. Funct. Mater. 2020, 30, 1905992.

[18] a) W. Chen, Q. Xiang, T. Peng, C. Song, W. Shang, T. Deng, J. Wu, iScience 2020, 23, 101532; b) K. J. J. Mayrhofer, D. Strmcnik, B. B. Blizanac, V. R. Stamenkovic, M. Arenz, N. M. Markovic, Electrochim. Acta 2008, 53, 3181.

[19] A.J. Bard, L. R. Faulkner, Electrochemical methods and applications, Wiley-Interscience, New York, London, 2000.

[20] R. Chen, H. Li, D. Chu, G. Wang, J. Phys. Chem. C 2009, 113, 20689.

[21] M. A. Kazakova, D. M. Morales, C. Andronescu, K. Elumeeva, A. G. Selyutin, A. V. Ishchenko, G. V. Golubtsov, S. Dieckhöfer, W. Schuhmann, J. Masa, Catal. Today 2020, 357, 259.
[22] a) L. Köhler, L. Szabadics, C. Jooss, M. Risch, Batteries \& Supercaps 2019, 2 , 364; b) V. Celorrio, A. S. Leach, H. Huang, S. Hayama, A. Freeman, D. W. Inwood, D. J. Fermin, A. E. Russell, ACS Catal. 2021, 6431.

[23] M. Risch, K. A. Stoerzinger, B. Han, T. Z. Regier, D. Peak, S. Y. Sayed, C. Wei, Z. J. Xu, Y. Shao-Horn, J. Phys. Chem. C 2017, 121, 17682.

[24] R. Qiao, T. Chin, S. J. Harris, S. Yan, W. Yang, Curr. Appl. Phys. 2013, 13, 544.

[25] R. K. Hocking, R. Brimblecombe, L.-Y. Chang, A. Singh, M. H. Cheah, C. Glover, W. H. Casey, L. Spiccia, Nat. Chem. 2011, 3, 461.

[26] a) T. Takashima, K. Hashimoto, R. Nakamura, J. Am. Chem. Soc. 2012, 134, 1519; b) H. Ooka, T. Takashima, A. Yamaguchi, T. Hayashi, R. Nakamura, Chem. Commun. 2017, 53, 7149.

[27] R. Ignatans, G. Mallia, E. A. Ahmad, L. Spillane, K. A. Stoerzinger, Y. ShaoHorn, N. M. Harrison, V. Tileli, J. Phys. Chem. C 2019, 123, 11621.

[28] A. S. Ryabova, F. S. Napolskiy, T. Poux, S. Y. Istomin, A. Bonnefont, D. M. Antipin, A. Y. Baranchikov, E. E. Levin, A. M. Abakumov, G. Kéranguéven et al., Electrochim. Acta 2016, 187, 161.

[29] K. A. Stoerzinger, M. Risch, B. Han, Y. Shao-Horn, ACS Catal. 2015, 5, 6021.

Manuscript received: May 31, 2021

Revised manuscript received: July 21, 2021

Accepted manuscript online: July 28, 2021 